\title{
Cases with imaging MRI
}

\section{Georgios Fragkiadakis, ENT Consultant Royal Lancaster Infirmary}

13 year old girl admitted with a sudden onset of left sided facial and retroorbital pain, nausea and visual disturbances and reduction in the peripheral visual fields. Seen by ophthalmology and diagnosed with left optic neuritis. Had MRI and a left sphenoidal mucocele was identified. Started treatment with steroid and antibiotics for 24 hours. No improvements, visual fields worse the second day of treatment. Had CT sinuses. Decision to be taken to theatre and under general anaesthetic she had a left sphenoidotomy and marsupulisation of the mucocele. Clinically it was a mucocele/not mucopyocele. Uneventful recovery. Clinically started improving gradually, with improvement in headache, visual acuity and visual fields and was discharged few days later on oral antibiotics and

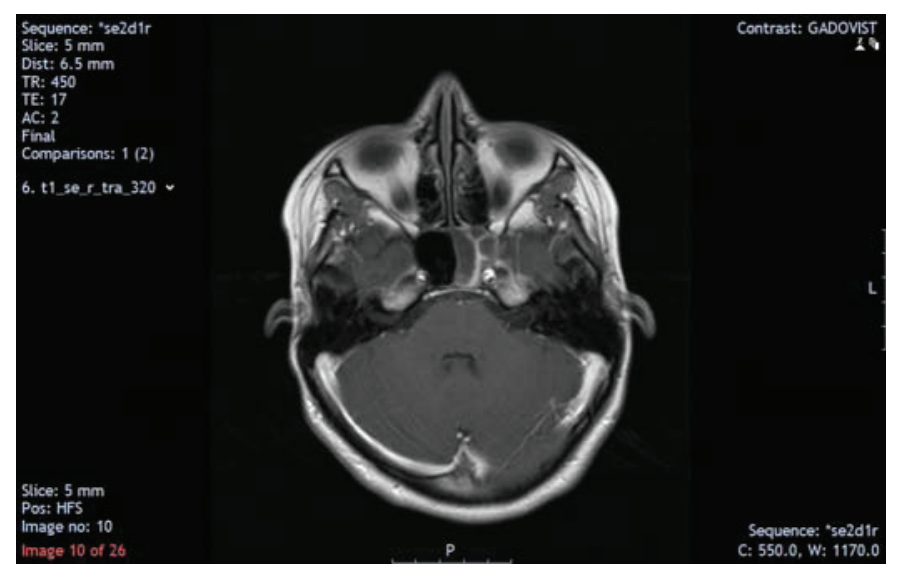

74 year old male who presented with fever, dyspnoea, mild stridor and trismus. Had an initial CT (small abscess seen) then taken to theatre for EUA throat and USS guided aspiration of a right deep neck abscess. Started on steroids and cefuroxime and metronidazole. Transferred to ITU for observation. The aspirate from the neck grew anaerobes. After almost a week, no improvement, neck collection increasing, had an MRI which showed diffuse multi lobulated deep neck abscess. Taken to theatre and under general anaesthetic he had I and D of a right parapharyngeal/parotid and submandibular abscess. Most

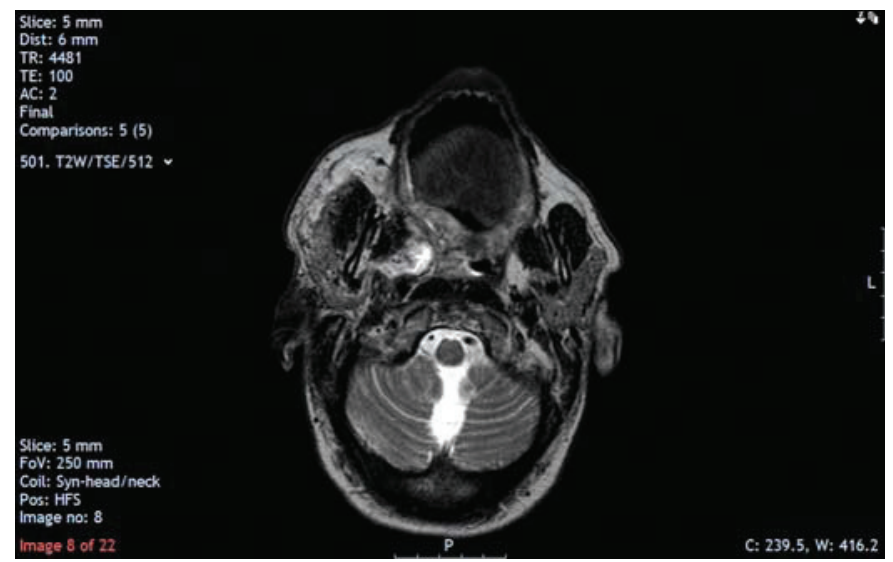

tapering dose of steroids. Eventually all symptoms resolved and vision became normal.

Had similar episode about a month after the initial episode. Seen in A\&E, another MRI with the suspicion of recurrence of sphenoiditis/mucopyocele, MRI clear. No further surgery. Had antibiotics and steroids and again complete resolution of symptoms.

The image on the left is the pre-op appearance and the one on the right the post op, the second time she was admitted with the same symptoms (but not as severe). So as per post op notes, she didn't have a mucopyocele that would definitely explain her optic neuritis. Rhinology MDT discussion was of the opinion that this was the correct management.

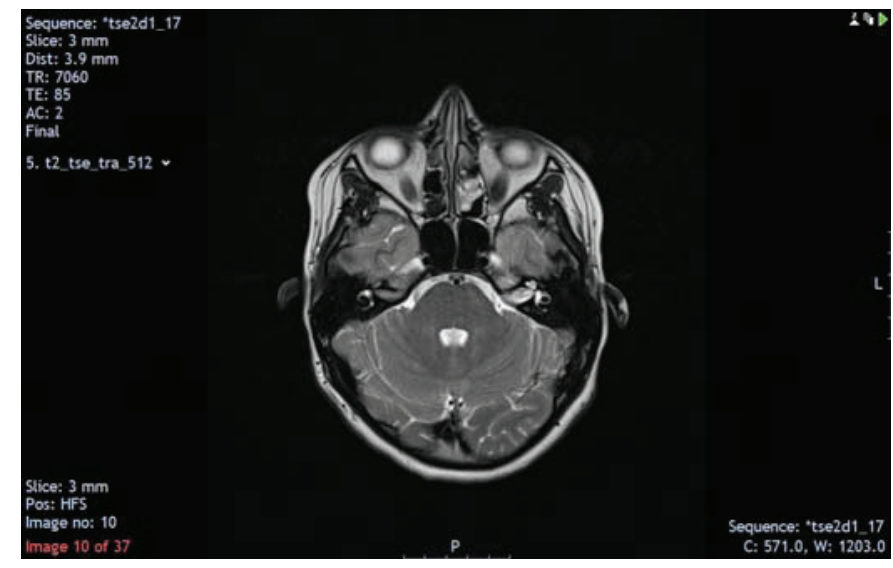

likely it was peritonsillar or dental in origin. A week later he had tonsillectomy and washout of the deep neck abscess. Histology was clear of any malignancy, but actinomyces were identified in the histology specimen. The patient recovered completely and was discharged 18 days after admission. The right image is a $\mathrm{T} 2$ sequence with heterogeneous appearances in the right parapharyngeal space, whereas in the left side it is a T1 sequence with gadolinium, which shows the peripheral enhancement of the multi lobulated abscess.

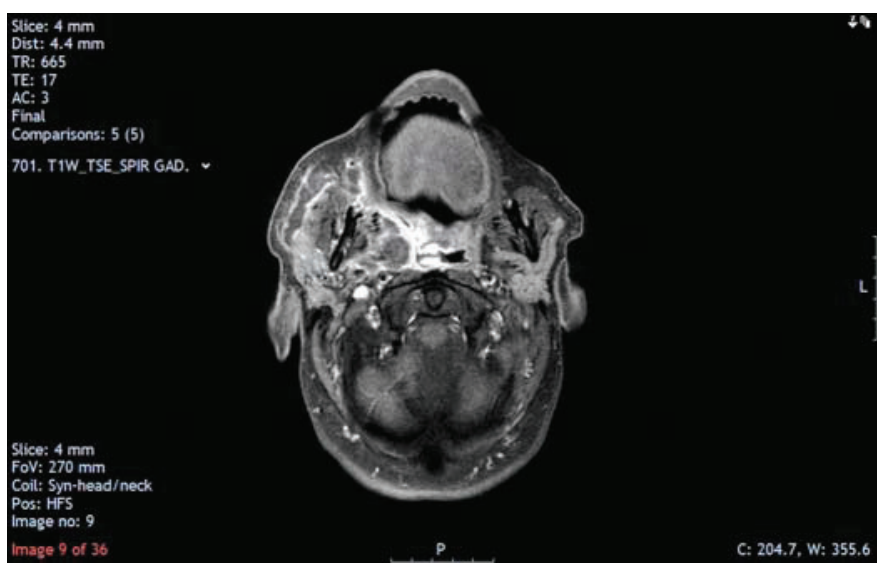

Correspondence to: Georgios.Fragkiadakis@mbht.nhs.uk 